\title{
Le travail curriculaire des enseignants en éducation physique : problématique de recherche et cadre théorique
}

\section{HRONIQUE • Recherche étudiante}

\section{Résumé Problématique et contextualisation}

Ce texte vise à présenter la problématique, quelques éléments du cadre théorique et nos questions de recherche sur le travail curriculaire des enseignants, dans le contexte de mise en œuvre d'un nouveau curriculum en éducation physique (NCÉP) dans les écoles de Cuiabá, au Brésil.

\section{Mots-clés}

Curriculum, mise en œuvre, enseignants, travail curriculaire, éducation physique

Abstract

This text aims to present the problematic, some elements of the theoretical

framework and our research questions on the curricular work of teachers, in the context of the implementation of a new curriculum in physical education (NCPE) in the Schools of Cuiabá, in Brazil.

Keywords

Curriculum, implementation, teachers, curriculum work, physical education
Au cours des dernières décennies, il y a eu des réformes curriculaires dans différents pays, à l'échelle mondiale. Cependant, bien que les changements aient lieu sur le plan des réformes et des politiques, on ne peut en dire autant sur le terrain. En effet, changer un curriculum est une entreprise complexe partout dans le monde (Fullan, 2001).

Dans le contexte des écoles municipales de Cuiabá, dans l'État de Mato Grosso, au Brésil, un nouveau curriculum d'éducation physique (NCÉP) a été mis en œuvre en 2012, dont les contenus sont basés sur les jeux, les sports, les danses, les gymnastiques, les luttes et les connaissances sur le corps. Par ailleurs, le Département municipal de l'éducation a offert un programme de formation continue pour soutenir la réforme. Malgré tout, nous en savons très peu sur l'impact du NCÉP sur les pratiques enseignantes. De plus, il existe peu d'études empiriques sur le curriculum réellement enseigné en éducation physique (ÉP) (Betti, Ferraz et Dantas, 2011; Lenzen, 2012).

\section{Cadre théorique}

Différents auteurs proposent des modèles ou identifient différents niveaux pour interpréter la mise en œuvre du curriculum. En ce sens, Perrenoud (1993) distingue les curriculums prescrit, réel et caché. Briot (1999) propose trois références curriculaires :la macrostructurale, issue des curriculums officiels; la mésostructurelle, issue du projet pédagogique de l'école ou de la formation continue; et la microstructurelle, issue des planifications des enseignants. Dans 
son modèle, Gimeno Sacristán (2000) différencie quant à lui les curriculums : prescrit, présenté aux enseignants, façonné par les enseignants, en action, réalisé et évalué.

Dans notre étude, nous retenons davantage le concept clé de « travail curriculaire » issu de la sociologie du travail enseignant. À notre avis, cette approche met un accent important sur le rôle de l'enseignant en tant que protagoniste du curriculum.

Dans cette perspective, Tardif et Lessard (1999) expliquent que le travail curriculaire des enseignants est un cycle continu d'adaptation et de transformation du curriculum officiel. Cette adaptation se développe en fonction des contraintes situationnelles, des ressources disponibles, de l'expérience antérieure des enseignants, de leurs préférences et valeurs, de leur compréhension de la matière, de leur interprétation des besoins des élèves, etc. Le travail curriculaire comprend les phases de planification, de l'enseignement en classe et de l'évaluation. En outre, il existe : a) des facteurs personnels, b) des facteurs internes à la situation de l'enseignement et c) des facteurs externes à l'enseignement influençant le travail curriculaire (voir figure 1).

Notre démarche s'appuie aussi sur le cadre de l'ergonomie française et ses notions de travail prescrit et réel, nous mobilisons également les concepts de prescriptions descendantes venant de la structure organisationnelle (dans notre cas, du ministère de l'Éducation du Brésil ou du Département municipal de l'éducation de Cuiabá) et de prescriptions remontantes, venant des collectifs de travail (dans notre cas, des projets pédagogiques des écoles) (Six, 1999, cité dans Daniellou, 2002).

Dans la figure suivante, nous présentons les différents facteurs qui ont une influence sur le travail curriculaire, ainsi que les prescriptions descendantes et remontantes qui peuvent guider les activités de planification de l'enseignant, l'enseignement en classe et l'évaluation, c'est-à-dire le travail d'adaptation et de transformation du curriculum par les enseignants.

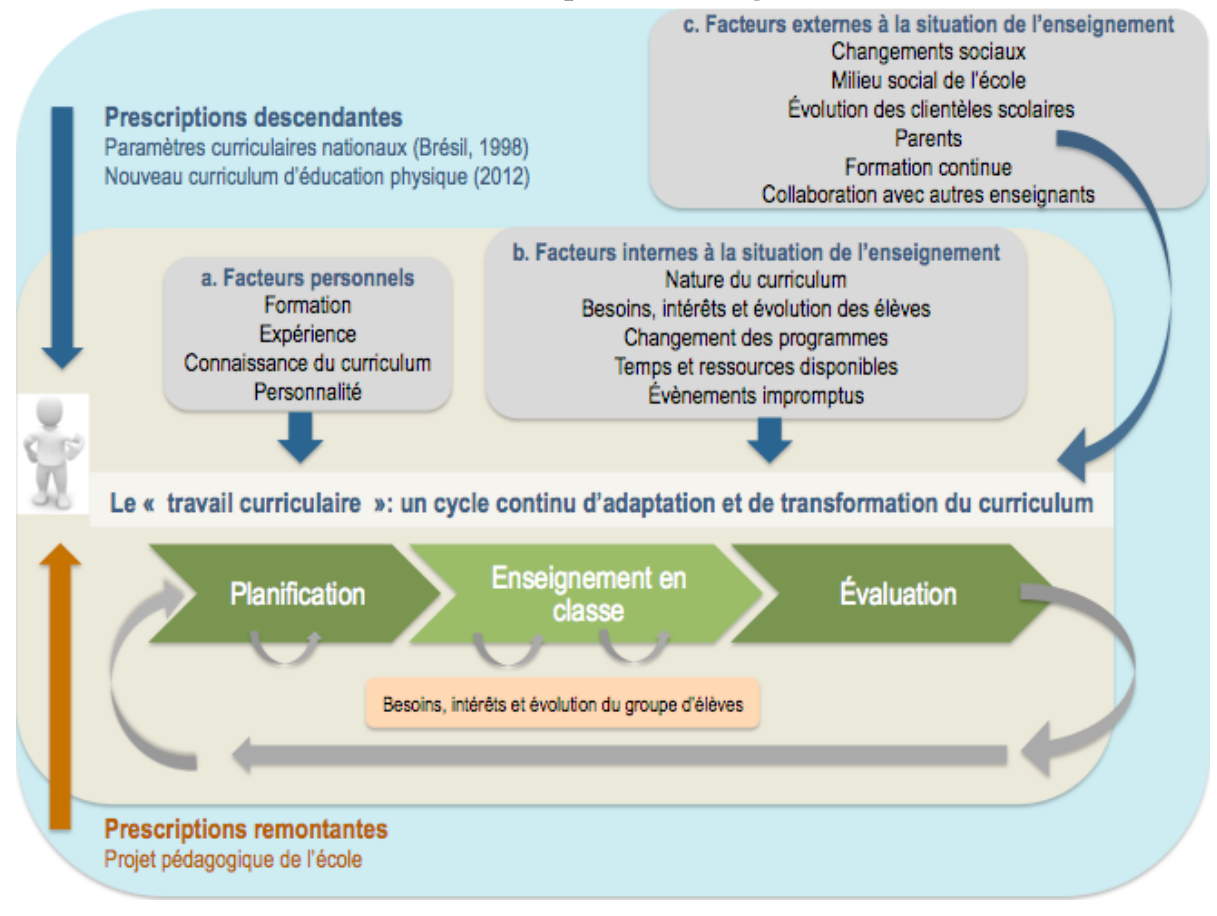

Figure 1 - Schéma de l'objet d'étude 
Il est important de noter que le travail curriculaire commence lors de la préparation des cours. À ce moment-là, les enseignants s'approprient et interprètent le curriculum officiel, lequel est alors adapté en fonction de la situation d'enseignement anticipée (Borges et Lessard, 2008). Cependant, lors de l'action en salle de classe, les choses se passent rarement comme prévu. Ainsi, même si la planification est minutieuse, les enseignants doivent la modifier au cours de l'année, des étapes et des journées. En outre, l'évaluation ne se limite pas à mesurer l'apprentissage des élèves, mais correspond aussi aux différents jugements que l'enseignant porte sur sa pratique, en apportant des ajustements à la planification afin de l'adapter et de la transformer en fonction du curriculum réel (Tardif et Lessard, 1999).

\section{Questions de recherche}

En considérant cette problématique et à la lumière de notre cadre théorique, nous nous intéressons à la complexité du travail des enseignants d'ÉP à Cuiabá, notamment en ce qui concerne le travail curriculaire dans le contexte de la mise en œuvre du NCÉP. Ainsi, nous formulons notre question générale de recherche comme suit : comment les enseignants s'approprient-ils le NCÉP dans leur travail quotidien? Autrement dit, comment réalisent-ils et façonnent-ils le curriculum prescrit?

Plus spécifiquement, nous nous posons les questions suivantes: quelles sont les références curriculaires (prescrites) qui guident le travail des enseignants d'ÉP? Quel est le rôle du contexte scolaire dans ce processus? Comment les enseignants abordent-ils leur travail curriculaire et la tension entre le curriculum prescrit et les contraintes contextuelles?

En bref, nous souhaitons contribuer à l'amélioration des politiques et pratiques curriculaires, en considérant les points de vue des acteurs qui se trouvent sur la ligne de front du processus éducatif, c'est-à-dire les enseignants.

\section{Références}

Betti, M., Ferraz, O. L. et Dantas, L. E. P. B. T. (2011). Educação Física escolar: estado da arte e direções futuras. Revista Brasileira de Educaçao Fisica e Esporte, 25, 105-115. http://dx.doi.org/10.1590/s1807-55092011000500011

Borges, C. et Lessard, C. (2008). Pratique enseignante et travail curriculaire dans le contexte de la réforme du secondaire au Québec. Les sciences de l'éducation pour l'Ére nouvelle, 41(4), 29-57. http://dx.doi.org/10.3917/1sdle.414.0029

Briot, M. (1999). Les stratégies des enseignants d'ÉPS dans le choix de leurs contenus d'enseignement. Revue française de pédagogie, 129(1), 73-85. http://dx.doi.org/10.3406/rfp.1999.1066

Daniellou, F. (2002). Le travail des prescriptions. Dans Congrès de la SELF, 37, Les évolutions de la prescription. Actes... (p. 916). Repéré à http://ergonomie-self.org/wp-content/uploads/2016/01/congres-self-2002-aix-daniellou-travail-prescriptions.pdf

Fullan, M. (2001). The new meaning of educational change (3e éd.). New York, NY : Teachers College Press.

Gimeno Sacristán, J. G. (2000). O currículo: uma reflexão sobre a prática (3éd.). Porto Alegre : Artmed.

Lenzen, B. (2012). Les activités curriculaires des enseignants d'ÉPS, entre prescription et liberté : une revue de littérature. e-Journal de la recherche sur l'intervention en éducation physique et sport, 27, 27-65. Repéré à https://archive-ouverte.unige.ch/unige:26251 
Perrenoud, P. (1993). Curriculum : le formel, le réel, le caché. Dans J. Houssaye (dir.), La pédagogie : une encyclopédie pour aujourd'bui (p. 61-76). Paris : ESF.

Tardif, M. et Lessard, C. (1999). Le travail enseignant au quotidien : contribution à l'étude du travail dans les métiers et les professions d'interactions humaines. Québec, QC : Presses de l'Université Laval.

\section{Note de bas de page}

1 Boursier du CAPES

\section{Pour citer cet article}

Godoi, M. (2016). Le travail curriculaire des enseignants en éducation physique : problématique de recherche et cadre théorique. Formation et profession. 24(3), 79-82. http://dx.doi.org/10.18162/fp.2016.a103 